\title{
Corrigendum: Previous Radiotherapy Increases the Efficacy of IL-2 in Malignant Pleural Effusion: Potential Evidence of a Radio-Memory Effect?
}

\author{
Dawei Chen ${ }^{1}$, Xinyu Song ${ }^{2,3}$, Haiyong Wang ${ }^{2}$, Zhenwu Gao ${ }^{4}$, Wenjuan Meng ${ }^{5}$, \\ Shuquan Chen ${ }^{6}$, Yunfeng $\mathrm{Ma}^{7}$, Youda Wang ${ }^{8}$, Kong $\mathrm{Li}^{1}$, Jinming $\mathrm{Yu}^{{ }^{1 *}}$ and Jinbo Yue ${ }^{1 \star}$ \\ ${ }^{1}$ Department of Radiation Oncology, Shandong Cancer Hospital affiliated to Shandong University, Jinan, China, \\ ${ }^{2}$ Department of Internal Medicine-Oncology, Shandong Cancer Hospital affiliated to Shandong University, Jinan, China, \\ ${ }^{3}$ School of Medicine and Life Sciences, University of Jinan-Shandong Academy of Medical Sciences, Jinan, China, \\ ${ }^{4}$ Department of oncology, Affiliated Hospital of Weifang Medical University, Weifang, China, ${ }^{5}$ Weifang People's Hospital, \\ Weifang, China, ${ }^{6}$ Laiwu Hospital of Traditional Chinese Medicine, Laiwu, China, ${ }^{7}$ Laiwu People's Hospital, Laiwu, China, \\ ${ }^{8}$ Linyi City People's Hospital, Linyi, China
}

\section{OPEN ACCESS}

Edited and reviewed by: Patrik Andersson, Massachusetts General Hospital and Harvard Medical School, United States

*Correspondence: Jinming Yu sdyujinming@163.com Jinbo Yue yuejinbo@hotmail.com

Specialty section: This article was submitted to

Cancer Immunity and Immunotherapy, a section of the journal

Frontiers in Immunology

Received: 05 January 2021 Accepted: 05 March 2021 Published: 23 March 2021

Citation:

Chen D, Song $X$, Wang $H$, Gao $Z$, Meng W, Chen S, Ma Y, Wang Y, Li K, Yu J and Yue J (2021) Corrigendum: Previous Radiotherapy Increases the Efficacy of IL-2 in Malignant Pleural Effusion: Potential Evidence of a Radio-Memory Effect?

Front. Immunol. 12:649620. doi: 10.3389/fimmu.2021.649620
Keywords: non-small-cell lung cancer (NSCLC), radiotherapy, radio-memory effect, immunotherapy, interleukin-2 (IL-2), malignant pleural effusion (MPE)

\section{A Corrigendum on}

Previous Radiotherapy Increases the Efficacy of IL-2 in Malignant Pleural Effusion: Potential Evidence of a Radio-Memory Effect?

By Chen D, Song X, Wang H, Gao Z, Meng W, Chen S, Ma Y, Wang Y, Li K, Yu J, Yue J. (2018) Front. Immunol. 9:2916. doi: 10.3389/fimmu.2018.02916

In the original article, there were mistakes in Figure $\mathbf{1}$ and Tables 1-3 as published.

In Figure 1, instead of "354 with any prior RT", "288 with no prior RT", "324 with prior extracranial RT" and " 318 without prior extra-cranial RT", it should be "324 with any prior RT", "318 with no prior RT", "288 with prior extra-cranial RT” and “354 without prior extra-cranial RT", respectively.

Meanwhile, there were mistakes in Table $\mathbf{1}$ due to some incorrect statistical results. Consequently, Tables $\mathbf{2}$ and $\mathbf{3}$ also need to be revised as, after rechecking the data, ECOG score is not significant in Table 2 These errors were caused by the carelessness and mis-operation in statistics and have been identified by the authors so that this would not happen in the future. The corrected Figure 1 and Table 1 to 3 appear below.

Consequently, a correction has been made to "RESULTS", "Survival Outcomes": paragraphs 1 and 2:

"In univariate analysis of the 642 patients who received intrapleural IL-2, having had any prior radiotherapy $(\mathrm{p}=0.007)$ and having had extracranial radiotherapy $(\mathrm{p}=0.003)$ were associated with longer PFS. Multivariate analysis revealed that having had any radiotherapy and extracranial radiotherapy were independent predictors of PFS (Table 2).”

"In univariate analysis of the 642 patients who received intrapleural IL-2, having had any prior radiotherapy $(\mathrm{p}<0.001)$ and extracranial radiotherapy $(\mathrm{p}<0.001)$ were associated with longer OS. 
Multivariate analysis revealed that having had any radiotherapy and extracranial radiotherapy were independent predictors of OS (Table 3)."

The authors apologize for this error and state that this does not change the scientific conclusions of the article in any way. The original article has been updated.
Copyright (c) 2021 Chen, Song, Wang, Gao, Meng, Chen, Ma, Wang, Li, Yu and Yue. This is an open-access article distributed under the terms of the Creative Commons Attribution License (CC BY). The use, distribution or reproduction in other forums is permitted, provided the original author(s) and the copyright owner(s) are credited and that the original publication in this journal is cited, in accordance with accepted academic practice. No use, distribution or reproduction is permitted which does not comply with these terms.

3747 patients with MPE

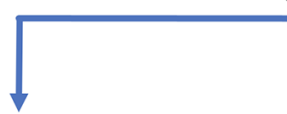

1506 patients treated

with intrapleural IL-2

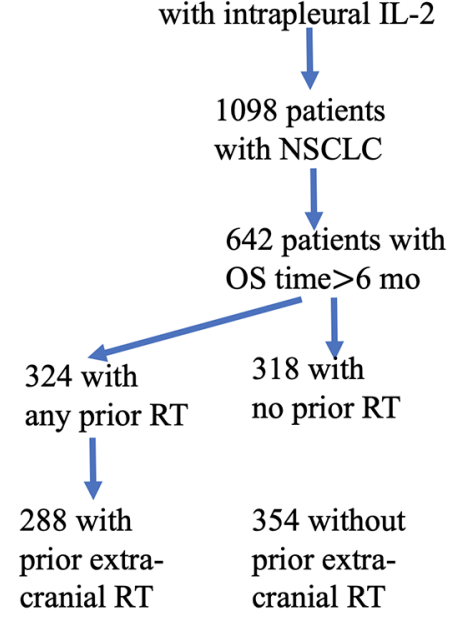

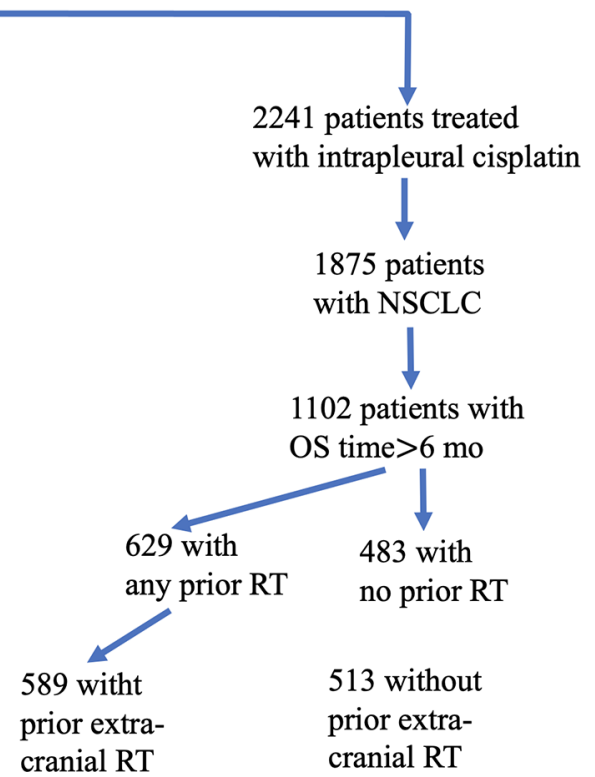

FIGURE 1 | Flowchart of eligible patients enrolled in this study. From a total of 3,747 patients with malignant pleural effusion (MPE), we identified 1,506 who had been treated with interpleural interleukin-2 (IL-2) and 2,241 who had been treated with intrapleural cisplatin. Of the 1,098 patients given IL-2 (and the 1,875 patients given cisplatin) who had non-small cell lung cancer, 642 who had received IL-2 survived for more than 6 months, and 1,102 who had received cisplatin survived for more than 6 months. Patients in each group were subdivided according to whether they had any vs. no radiotherapy (RT), or extracranial vs. no extracranial RT. 
TABLE 1 | Baseline characteristics.

\begin{tabular}{|c|c|c|c|c|c|c|c|}
\hline \multirow[t]{2}{*}{ Variable } & \multicolumn{4}{|c|}{ Previous radiotherapy } & \multicolumn{3}{|c|}{ Previous extracranial radiotherapy } \\
\hline & $\mathbf{N}$ & No $(n=318)$ & Yes $(n=324)$ & $p$ value & No $(n=354)$ & Yes $(n=288)$ & $p$ value \\
\hline \multicolumn{8}{|l|}{ Sex } \\
\hline Male & 360 & $168(53 \%)$ & $192(59 \%)$ & \multirow[t]{2}{*}{0.101} & $198(56 \%)$ & $162(56 \%)$ & \multirow[t]{2}{*}{0.936} \\
\hline Female & 282 & $150(47 \%)$ & $132(41 \%)$ & & $156(44 \%)$ & $126(44 \%)$ & \\
\hline \multicolumn{8}{|l|}{ Age, years } \\
\hline$\geq 55$ & 312 & $144(45 \%)$ & $168(52 \%)$ & \multirow[t]{2}{*}{0.497} & $174(49 \%)$ & $138(48 \%)$ & \multirow[t]{2}{*}{0.755} \\
\hline$<55$ & 330 & $174(55 \%)$ & $156(48 \%)$ & & $180(51 \%)$ & $150(52 \%)$ & \\
\hline \multicolumn{8}{|l|}{ ECOG PS Score } \\
\hline 0 & 122 & $60(19 \%)$ & $62(19 \%)$ & \multirow[t]{3}{*}{0.992} & $70(20 \%)$ & $52(18 \%)$ & \multirow[t]{3}{*}{0.859} \\
\hline 1 & 412 & $204(64 \%)$ & $208(64 \%)$ & & $225(63 \%)$ & $187(65 \%)$ & \\
\hline 2 & 108 & $54(17 \%)$ & $54(17 \%)$ & & $59(17 \%)$ & $49(17 \%)$ & \\
\hline \multicolumn{8}{|l|}{ Histopathological classification } \\
\hline Squamous cell & 198 & $90(28 \%)$ & $108(33 \%)$ & \multirow[t]{2}{*}{0.168} & $120(34 \%)$ & $78(27 \%)$ & \multirow[t]{2}{*}{0.063} \\
\hline Adenocarcinoma or other & 444 & $228(72 \%)$ & $216(67 \%)$ & & $234(66 \%)$ & $210(73 \%)$ & \\
\hline \multicolumn{8}{|l|}{ Smoking history } \\
\hline Never-smoker & 402 & $210(66 \%)$ & $192(59 \%)$ & \multirow[t]{2}{*}{0.076} & $233(66 \%)$ & $169(58 \%)$ & \multirow[t]{2}{*}{0.409} \\
\hline Former/current smoker & 240 & $108(34 \%)$ & $132(41 \%)$ & & $121(34 \%)$ & $119(42 \%)$ & \\
\hline \multicolumn{8}{|l|}{ Diagnosis method } \\
\hline CT guided biopsy & 264 & $126(40 \%)$ & $138(43 \%)$ & \multirow[t]{4}{*}{0.001} & $138(39 \%)$ & $126(44 \%)$ & 0.001 \\
\hline Pleural effusion cytology & 144 & $78(25 \%)$ & $66(20 \%)$ & & $90(25 \%)$ & $54(19 \%)$ & \\
\hline Thoracotomy & 174 & $72(23 \%)$ & $102(31 \%)$ & & $78(22 \%)$ & $96(33 \%)$ & \\
\hline Neck lymph node biopsy & 60 & $42(12 \%)$ & $18(6 \%)$ & & $48(14 \%)$ & $12(4 \%)$ & \\
\hline Color of Pleural Effusion & & & & & & & \\
\hline Bloody & 426 & $222(70 \%)$ & $204(63 \%)$ & 0.066 & $246(69 \%)$ & $180(63 \%)$ & 0.062 \\
\hline Light yellow & 216 & $96(30 \%)$ & $120(37 \%)$ & & $108(31 \%)$ & $108(37 \%)$ & \\
\hline Hematologic Findings & & & & & & & \\
\hline Neutrophil count, mean $\pm \mid \mathrm{QR}, \times 10^{3} / \mu \mathrm{|}$ & & $6.12 \pm 1.78$ & $4.48 \pm 1.34$ & 0.032 & $6.09 \pm 1.81$ & $4.4 \pm 1.32$ & 0.034 \\
\hline Total lymphocyte count, mean $\pm\left|\mathrm{QR}, \times 10^{3} / \mu\right|$ & & $1.34 \pm 0.35$ & $2.21 \pm 0.70$ & 0.021 & $1.35 \pm 0.36$ & $2.19 \pm 0.69$ & 0.022 \\
\hline Neutrophil-to-lymphocyte ratio & & $4.56 \pm 1.36$ & $2.06 \pm 0.70$ & $<0.01$ & $4.52 \pm 1.41$ & $2.02 \pm 0.75$ & $<0.01$ \\
\hline Intrapleural chemotherapy chemotherapy before II & & & & & & & \\
\hline Yes & 528 & $258(81 \%)$ & $270(83 \%)$ & 0.466 & $286(81 \%)$ & $242(84 \%)$ & 0.286 \\
\hline No & 114 & $60(19 \%)$ & $54(17 \%)$ & & $68(19 \%)$ & $46(19 \%)$ & \\
\hline History of brain metastases & & & $68(21 \%)$ & & $36(10 \%)$ & $32(11 \%)$ & \\
\hline No. Of previous systemic therapies, mean (range) & & $2(0-5)$ & $2(0-5)$ & 0.017 & $2(0-5)$ & $3(0-6)$ & 0.021 \\
\hline Previous systemic therapies before IL-2 & & & & & & & \\
\hline Yes & 486 & $236(74 \%)$ & $250(77 \%)$ & 0.384 & $259(73 \%)$ & $227(79 \%)$ & 0.097 \\
\hline No & 156 & $82(26 \%)$ & $74(23 \%)$ & & $95(27 \%)$ & $61(21 \%)$ & \\
\hline Radiotherapy schedule before distant metastasis & & & & & & & \\
\hline $\mathrm{ChT} \rightarrow \mathrm{CCRT}$ & & & $140(43 \%)$ & & & $140(49 \%)$ & \\
\hline $\mathrm{CCRT} \rightarrow \mathrm{ChT}$ & & & $61(19 \%)$ & & & $61(21 \%)$ & \\
\hline $\mathrm{ChT} \rightarrow \mathrm{RT}$ & & & $66(20 \%)$ & & & $66(23 \%)$ & \\
\hline CCRT alone & & & $21(7 \%)$ & & & $21(7 \%)$ & \\
\hline Intracranial radiotherapy & & & $36(11 \%)$ & & & 0 & \\
\hline Radiotherapy technology & & & & & & & \\
\hline Conventional radiotherapy & & & $164(\%)$ & & & $148(51 \%)$ & \\
\hline 3D-CRT/MRT & & & $160(\%)$ & & & $140(49 \%)$ & \\
\hline Previous SABR & & & & & & & \\
\hline Yes & 20 & & $20(\%)$ & & & $20(7 \%)$ & \\
\hline No & 622 & & $304(\%)$ & & & $268(93 \%)$ & \\
\hline
\end{tabular}

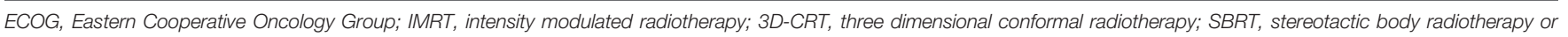
stereotactic radiosurgery. 
TABLE 2 | Predictors associated with progression free survival (PFS).

\begin{tabular}{|c|c|c|c|c|c|c|c|c|}
\hline & \multicolumn{2}{|c|}{$\mathrm{PFS}^{*}$} & \multicolumn{3}{|c|}{ Any previous RT and PFS† } & \multicolumn{3}{|c|}{ Previous extracranial RT and PFS $†$} \\
\hline $\begin{array}{l}\text { Sex } \\
\text { (Male vs Female) }\end{array}$ & 1.778 & 0.182 & & & & & & \\
\hline $\begin{array}{l}\text { Age } \\
\qquad(\geq 55 \text { vs }<55)\end{array}$ & 0.056 & 0.812 & & & & & & \\
\hline $\begin{array}{l}\text { ECOG PS Score } \\
(0 \text { vs } 1 \text { vs } 2)\end{array}$ & 0.104 & 0.747 & & & & & & \\
\hline $\begin{array}{l}\text { Smoking history } \\
\text { (Never vs Former/current) }\end{array}$ & 2.845 & 0.092 & 0.892 & $0.747-1.064$ & 0.204 & 0.862 & $0.721-1.029$ & 0.101 \\
\hline $\begin{array}{l}\text { Colour } \\
\text { (Bloody vs Yellow) }\end{array}$ & 0.178 & 0.673 & & & & & & \\
\hline $\begin{array}{l}\text { Any previous radiotherapy } \\
\text { (Yes vs No) }\end{array}$ & 7.299 & 0.007 & 0.805 & $0.677-0.957$ & 0.014 & & & \\
\hline $\begin{array}{l}\text { Previous extracranial radiotherapy } \\
\text { (Yes vs No) }\end{array}$ & 9.048 & 0.003 & & & & 0.752 & $0.632-0.895$ & 0.001 \\
\hline
\end{tabular}

Progression-free survival was defined as the time from the first administration of I.P. IL-2 to disease progression or death. HR, hazard ratio. *Univariate analysis.

${ }^{\dagger}$ Multivariate analysis.

TABLE 3 | Predictors associated with overall survival (OS)

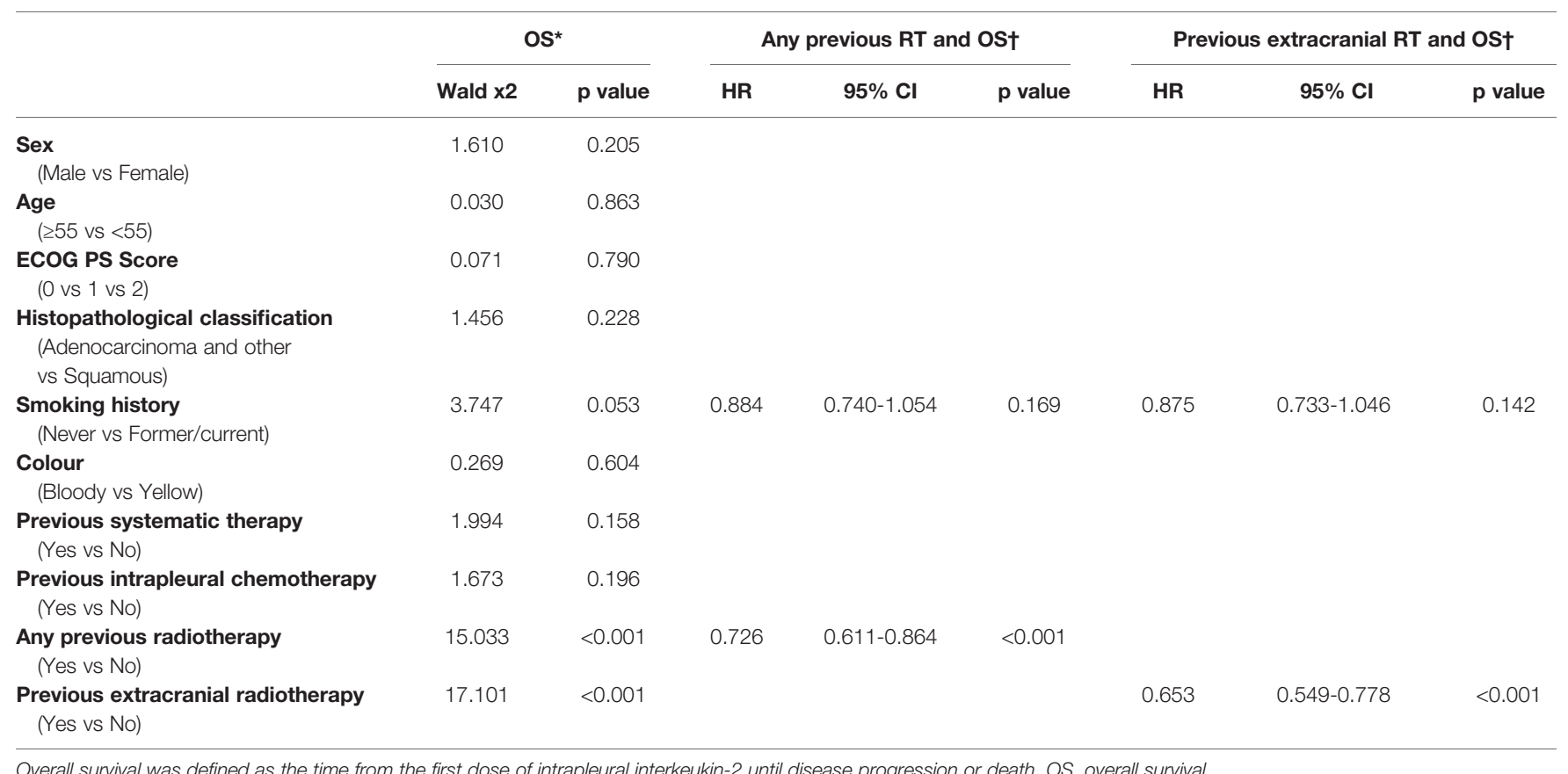

